\title{
Epidemic keratoconjunctivitis and chronic papillary conjunctivitis in London due to adenovirus type 19
}

\author{
S. DAROUGAR, M. P. QUINLAN, J. A. GIBSON, AND BARRIE R. JONES \\ From the Department of Clinical Ophthalmology, Institute of Ophthalmology and Moorfields Eye Hospital
}

D. A. McSWIGGAN

From the Public Health Laboratory and Department of Microbiology, Central Middlesex Hospital

SUMMARY Since July 1973 cases of keratoconjunctivitis resembling epidemic keratoconjunctivitis were observed in the External Eye Disease Clinic at Moorfields Eye Hospital, City Road, London. Adenovirus type 19 was isolated in human embryonic kidney cells from 21 patients. The majority were males between 20 and 40 years old. A small hospital outbreak involving six patients occurred.

Clinical features of the disease, consisting of moderate to severe follicular conjunctivitis, major subepithelial punctate keratitis, sometimes with pseudomembrane and scarring, were closely similar to those of epidemic keratoconjunctivitis caused by adenovirus type 8 . This similarity, as well as the ability of the agent to cause hospital outbreaks, indicates that adenovirus type 19 is a cause of epidemic keratoconjunctivitis.

A case of bilateral chronic papillary conjunctivitis that persisted for 16 months following an acute onset was described. Adeno 19 was isolated from the eye of the patient after 12 months of recrudescent or recurrent illness. Chronic adenovirus infection lacking the usual clinical picture of an acute follicular reaction has not hitherto been described. Such cases are probably important because of the obvious danger of continuing the carriage and shedding of infective adeno 19 from one outbreak to another, by presenting subsequently in eye clinics, and providing an un recognised source of infection to initiate further outbreaks of hospital transmission.

During the year 1973-74 it became apparent that an increase was occurring in the number of sporadic cases of acute keratoconjunctivitis with subepithelial punctate keratitis presenting in the External Eye Diseases Clinic, Moorfields Eye Hospital, London. Adenovirus type 19 (adeno 19) was isolated from a number of these cases. The epidemiological and clinical features of the disease appeared to be similar to those of epidemic keratoconjunctivitis (EKC) caused by adenovirus type 8 (adeno 8).

Adeno 19 was first isolated in 1955 from the eye of a Saudi Arabian child with active trachoma (Bell et al., 1960). Systemic infection by this serotype has been rare: in England and Wales during the period of 1969-72 only one isolate of adeno 19 was reported from the respiratory tract (Communicable Diseases Report, 1974). However, since the summer of 1973 several outbreaks, or sporadic cases, of adeno 19 keratoconjunctivitis have been reported in the USA (Hierholzer et al., 1974), Canada (Vas et al., 1974),

Address for reprints: Dr S. Darougar, Virus Laboratory, Institute of Ophthalmology, Judd Street, London WC1H $9 Q S$.
Belgium and Holland (Desmyter et al., 1974), and Scotland (Bell and Winton, 1975).

This paper presents the epidemiological and clinical features of adeno 19 keratoconjunctivitis observed in London and stresses the risks of hospital outbreaks of severe conjunctivitis with major punctate keratitis resembling EKC. It also records the isolation of adeno 19 from a chronic relapsing case of papillary conjunctivitis that would hitherto not have been suspected of being due to adenovirus, and so would offer considerable risk of initiating a new outbreak of hospital-based transmission of infection.

\section{Methods and materials}

CLINICAL EXAMINATION

The 21 patients in this study were selected on the basis of positive cultures of adeno 19 from among those referred to the External Eye Disease Clinic at Moorfields Eye Hospital for investigation of acute conjunctivitis. 
Symptoms and clinical signs observed with a Haag Streit 900 slit-lamp were recorded on a proforma using an extension of the method used for the study of trachoma (Dawson et al., 1975).

\section{Laboratory investigations}

\section{DIFFERENTIAL CYTOLOGY}

Conjunctival impressions were taken using a specially designed plastic spatula (Thatcher, Darougar, and Jones, in preparation). They were fixed with methanol, stained with Giemsa's method, mounted on glass slides, and examined microscopically.

\section{VIRUS ISOLATION}

Forty-two specimens were collected, by swabbing different areas of the conjunctiva with cotton wool swabs, from 21 patients. The swabs were placed in plastic capsules with 2SPS transport medium (Darougar and Jones, 1971) and stored in a liquid nitrogen refrigerator until transferred to a $-70^{\circ} \mathrm{C}$ refrigerator in the laboratory.

Each clinical specimen was inoculated into two tubes containing human embryonic kidney (HEK) cells and examined for the presence of cytopathic effect (CPE) up to 21 days (McSwiggan et al., 1975). The isolates were identified by the standard neutralisation test.

\section{SEROLOGY}

Serum collected from 15 of these patients by venepuncture at intervals of two to three weeks was tested by a complement fixation test (CFT) for adenovirus group antibody.

\section{Results}

SEX AND AGE DISTRIBUTION

The ratio of males to females was 15 to 6 . The ages of these patients ranged from 5 weeks to 59 years, but most were between 20 and 40 years old.

\section{SEASONAL VARIATION}

Of these 21 patients four were observed in June or July 1973, five between November 1973 and January 1974, 10 in June to August 1974, and two in October or November 1974. The highest incidence in June to August 1974 was partly due to an outbreak of cross-infection in the hospital. Several more cases of a similar nature were observed during this period, but no adenovirus was isolated from them.

\section{SOURCE OF INFECTION}

Of these 21 patients two had been in close contact with a friend who had been suffering from conjunctivitis, and'six became infected in hospital.
The other 13 had not been aware of contact with conjunctivitis or upper respiratory infection in families, friends, or workmates, nor had they attended hospitals or clinics or visited a swimming pool.

\section{HOSPITAL OUTBREAK}

In the early summer of 1974 cross-infection occurred at Moorfields Eye Hospital (Fig. 1). A 5-week-old baby who had been attending with TRIC ophthalmia neonatorum, from which Chlamydia trachomatis was isolated, apparently contracted adenovirus infection in the Clinic. Four days after a clinical examination the baby developed a moderately severe exacerbation of inflammation in both eyes. The mother of this baby and his $2 \frac{1}{2}$-year-old sister each developed conjunctivitis, seven and nine days respectively, after the onset of disease in the baby. Adeno 19 was isolated from the eyes of all three patients.

Another ophthalmologist in the clinic apparently contracted adenovirus infection from a close friend who had been suffering from a severe conjunctivitis with subepithelial keratitis suggestive of an adenovirus infection. While he was incubating the infection, he examined a 16-year-old girl who had teen attending the clinic for dry eyes. Six days later she developed adenovirus infection of the eye. Two other members of her family - a $2 \frac{1}{2}$-year-old boy and his mothercontracted the infection nine and 21 days respectively from the onset of her conjunctivitis. Adeno 19 was isolated from the eyes of the doctor and three members of this family. Four other patients apparently contracted the infection from this doctor, but the culture tests on these patients were negative.

INCUBATION PERIOD

In the hospital outbreak the incubation period ranged between four and 12 days with a mean of eight.

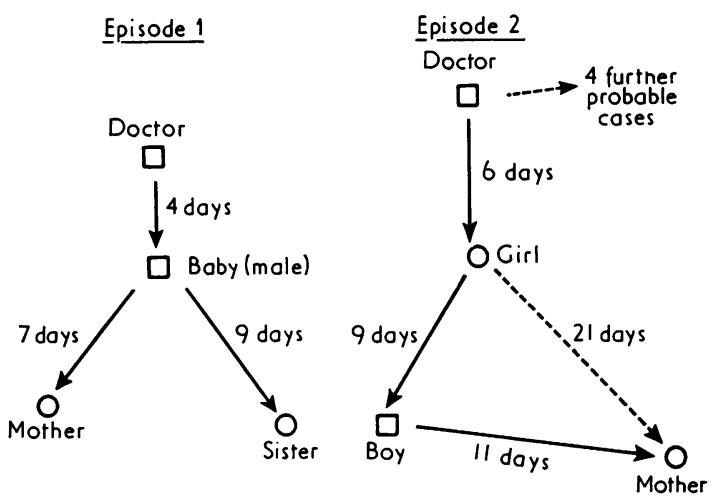

Fig. 1 Transmission of infection by adeno 19 in two episodes of hospital cross-infection 


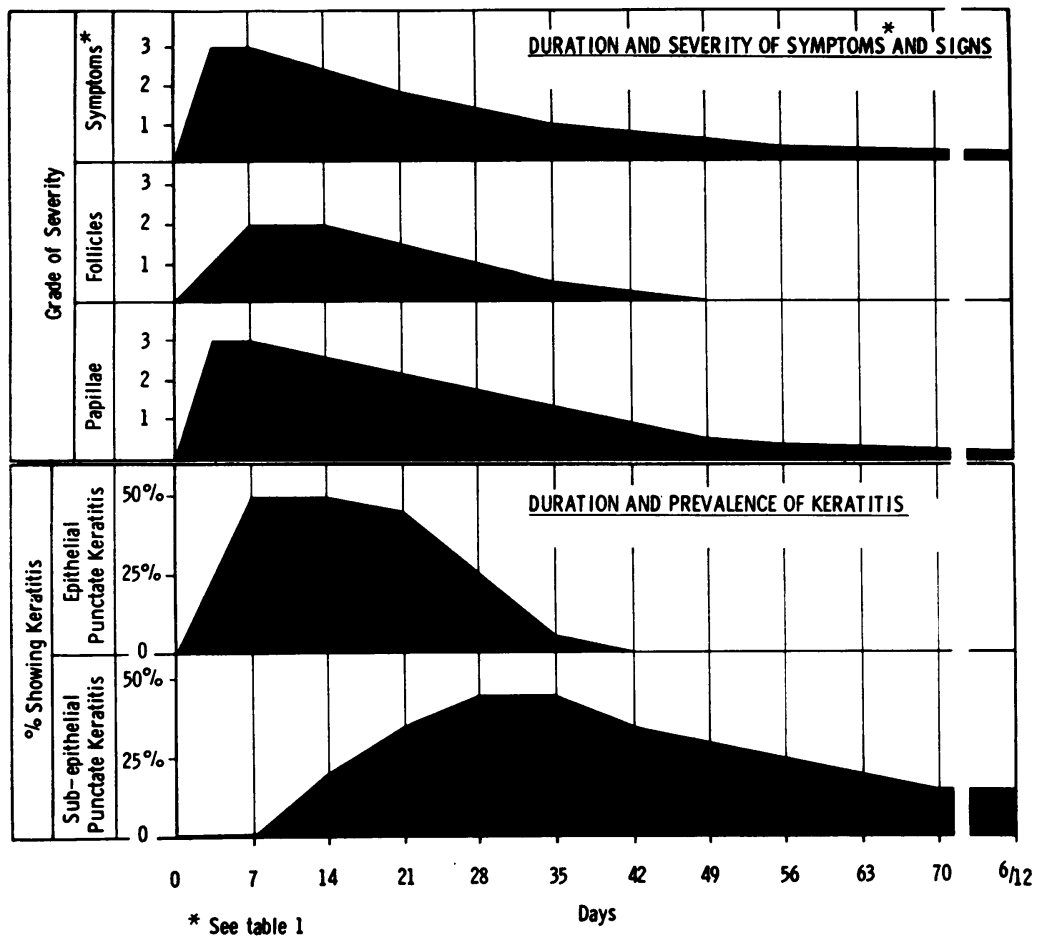

Fig. 2 Duration and severity of symptoms and signs of disease in 21 cases of adeno 19 keratoconjunctivitis

SYSTEMIC INFECTION

Four of the 21 patients had rhinitis and sore throats for one week before the conjunctivitis, and one complained of general malaise.

\section{LYMPHADENOPATHY}

Three of the 21 patients had enlarged preauricular nodes at the first visit. In two patients this was unilateral and lasted only one week. In the third patient bilateral preauricular adenopathy persisted for two weeks. No glands were visibly enlarged, and no submandibular or cervical lymphadenopathy was observed.

\section{SYMPTOMS}

All patients complained of moderate to severe lachrymation, discharge, swelling, inflammation, and a sensation of the presence of a foreign body in the affected eye during the early stage of the infection (Table 1, Fig. 2).

Symptoms were milder in the younger children and in patients who first attended more than 10 days after the onset of symptoms.

SIGNS

In 10 of the 21 cases the second eye became inflamed within four days of the onset of the infection. This occurred between the fifth and tenth day in a further six cases. Five remained unilateral. The average duration of conjunctival inflammation was six weeks (range three to 16 months).

The bulbar conjunctiva showed moderate to severe hyperaemia in 14 and mild to moderate chemosis in 10 patients, with ecchymosis in six.

The prevalence and severity of clinical signs in the palpebral conjunctiva are shown in Table 2 and Fig. 2. At the first attendance all the patients except two who first attended during the resolving stage of their infection showed moderate to severe hyperaemia and diffuse infiltration with papillary and follicular reactions.

Papillary reaction was most severe in the upper tarsal conjunctiva (Figs. 3 and 4). It persisted for

Table 1 Prevalence and severity of symptoms at first visit in 21 cases of adeno 19 keratoconjunctivitis

\begin{tabular}{|c|c|c|c|}
\hline \multirow{2}{*}{ Symptoms } & \multicolumn{3}{|c|}{ Severity } \\
\hline & Mild & Moderate & Severe \\
\hline Lachrymation & 5 & 10 & 6 \\
\hline Discharge & 5 & 5 & 6 \\
\hline Swelling of the lids & 5 & 6 & 4 \\
\hline Redness & 5 & 10 & 5 \\
\hline Itching & 5 & 2 & 1 \\
\hline Grittiness & 9 & 5 & 0 \\
\hline Pain & 3 & 1 & 1 \\
\hline Photophobia & 7 & 3 & 1 \\
\hline Blurred vision & 6 & 2 & $\mathbf{0}$ \\
\hline
\end{tabular}




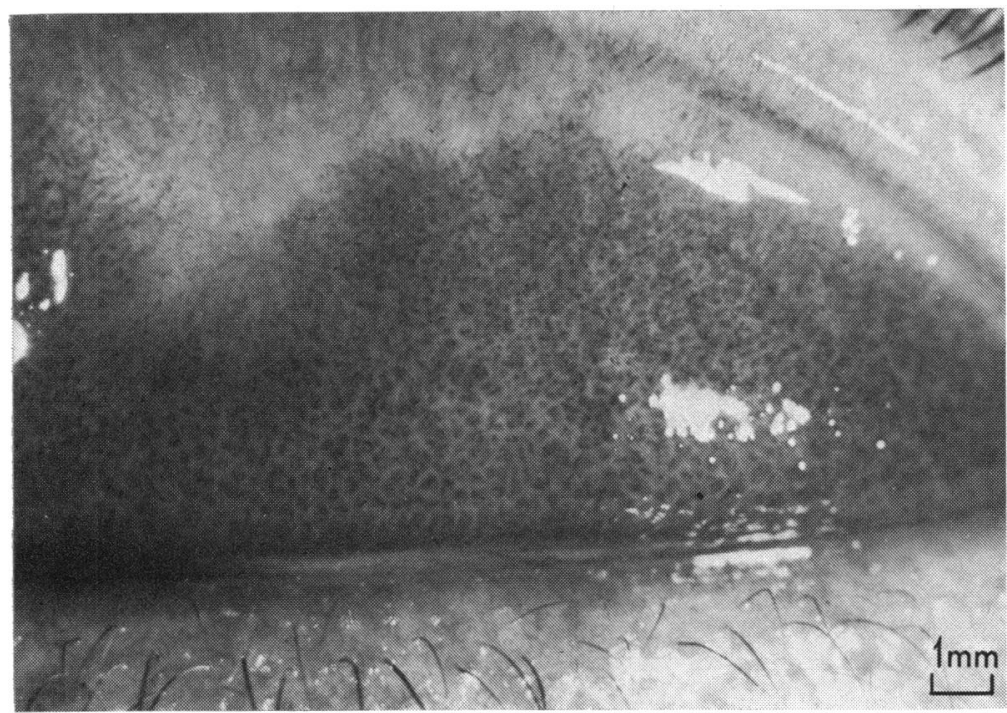

Fig. 3 Severe papillary reaction in upper tarsal conjunctiva partially obscured by early pseudomembrane in the first week of type 19 adenovirus conjunctivitis $(\times 6)$

about six weeks in 18 patients, three months in two, and for 16 months in one (Fig. 2).

Table 2 Prevalence and severity of signs in the palpebral conjunctiva at first visit in 21 cases of adeno 19 keratoconjunctivitis

\begin{tabular}{|c|c|c|c|}
\hline \multirow{2}{*}{ Signs } & \multicolumn{3}{|c|}{ Severity } \\
\hline & Mild & Moderate & Severe \\
\hline Hyperaemia & 2 & 11 & 8 \\
\hline Diffuse infiltration & 3 & 15 & 3 \\
\hline Papillary hypertrophy & 0 & 8 & 13 \\
\hline Follicular hypertrophy & 4 & 12 & 5 \\
\hline Pseudomembrane & 3 & 6 & 0 \\
\hline Scar & 2 & 2 & 0 \\
\hline
\end{tabular}

Moderate follicular reaction was present, mainly in the upper and lower fornices, in all 21 patients (Figs. 5, 6, and 7). The follicles were rather small, discrete and lasted three to seven weeks (Fig. 2).

Pseudomembrane (Figs. 8, 9, and 10) was observed in nine of the patients with severe conjunctivitis. It developed seven to $\mathbf{1 0}$ days after the onset of infection and lasted one to two weeks. Mild or moderate linear or diffuse scarring (Figs. 11, 12, and 13) of the conjunctiva developed 20 to 30 days from the onset of the infection in four patients who had had rather well marked pseudomembrane.

One patient was of particular interest. In July 1973 a girl of 24 developed an acute bilateral

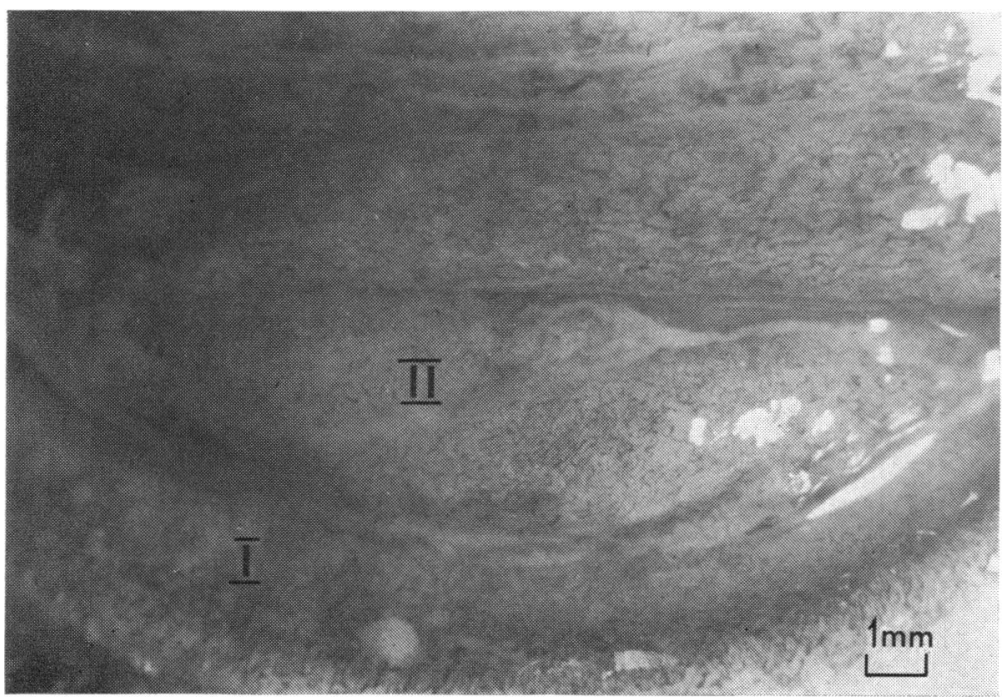

Fig. 4 Papillary hyperplasia in lower lid conjunctiva: (I) tarsal, (II) fornix 


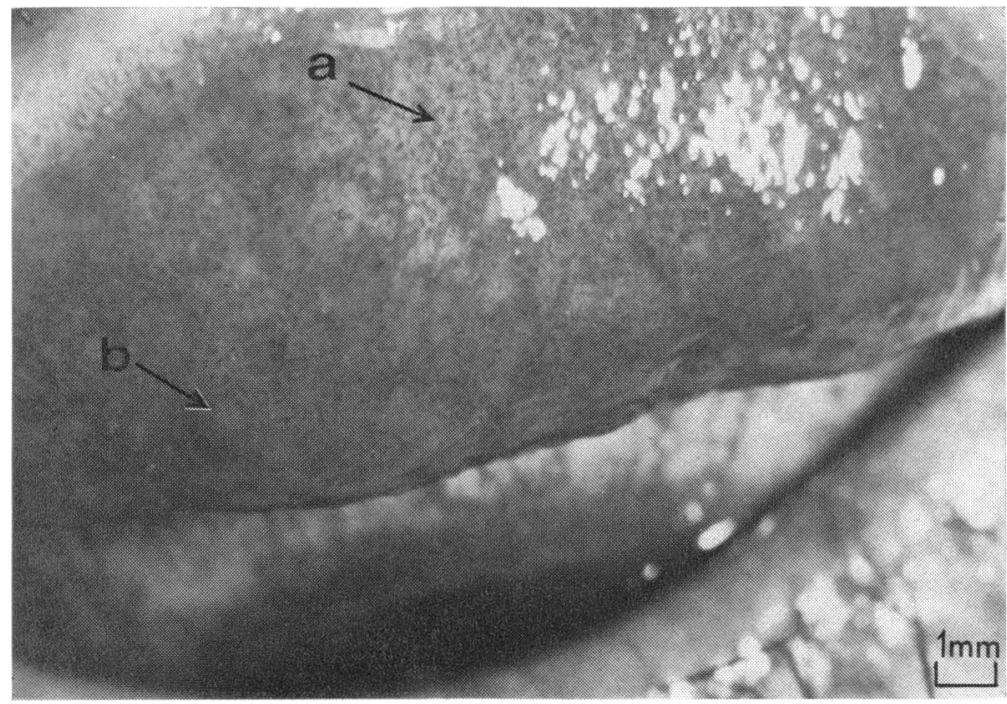

Fig. 5. Mixed papillary (a) and follicular reaction (b) in upper tarsal conjunctiva in the second week of type 19 adenovirus conjunctivitis

keratoconjunctivitis of six weeks' duration. After this she suffered from chronic conjunctivitis with several recrudescences of acute inflammation in one or other eye. She was found to have a bilateral chronic papillary conjunctivitis, most marked in the upper tarsal area, and her cornea were clear. Adeno 19 was isolated from the conjunctiva, when she was referred for investigation, 12 months after the onset of her infection. The condition persisted for a further four months. But after a course of topical treatment with $1 \%$ trifluorothymidine (TFT) eye-drops the patient was relieved of symptoms, and the signs of conjunctival inflammation disappeared.

\section{KERATITIS}

Of the 21 patients 15 developed keratitis. Moderate to severe fine epithelial punctate keratitis (Jones, 1962) was observed in 10 patients, mainly three to four days from the onset of the conjunctivitis and lasting for one to four weeks. Coarse epithelial punctate keratitis (Jones, 1962) was observed in nine patients, generally in association with fine epithelial punctate keratitis. Thirteen patients

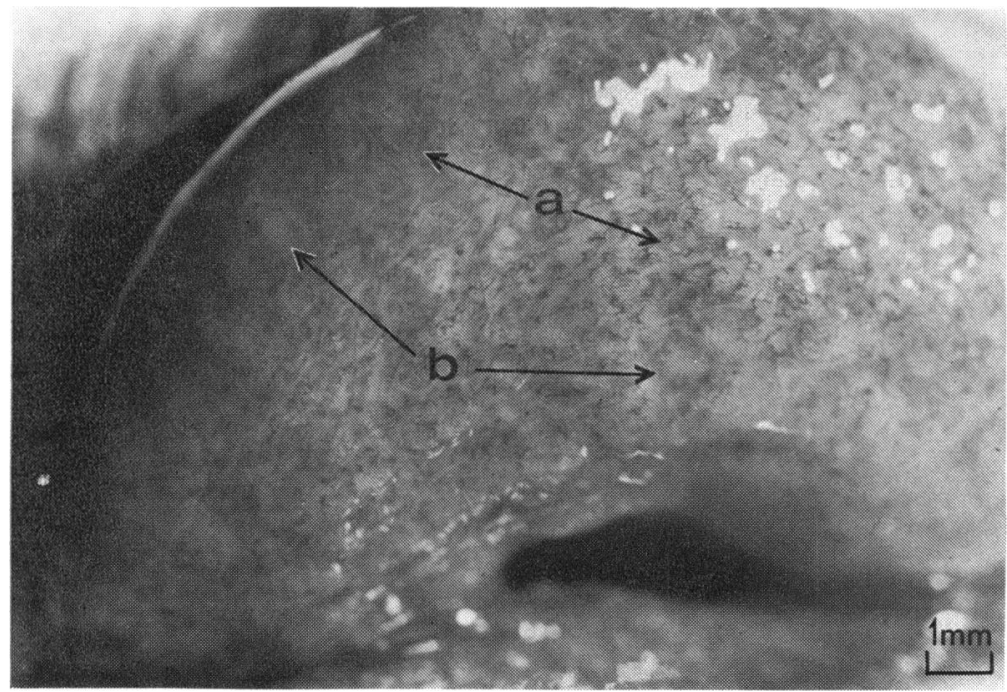

Fig. 6 Mixed papillary (a) and follicular reaction (b) in upper fornix conjunctiva in the second week of type 19 adenovirus conjunctivitis 


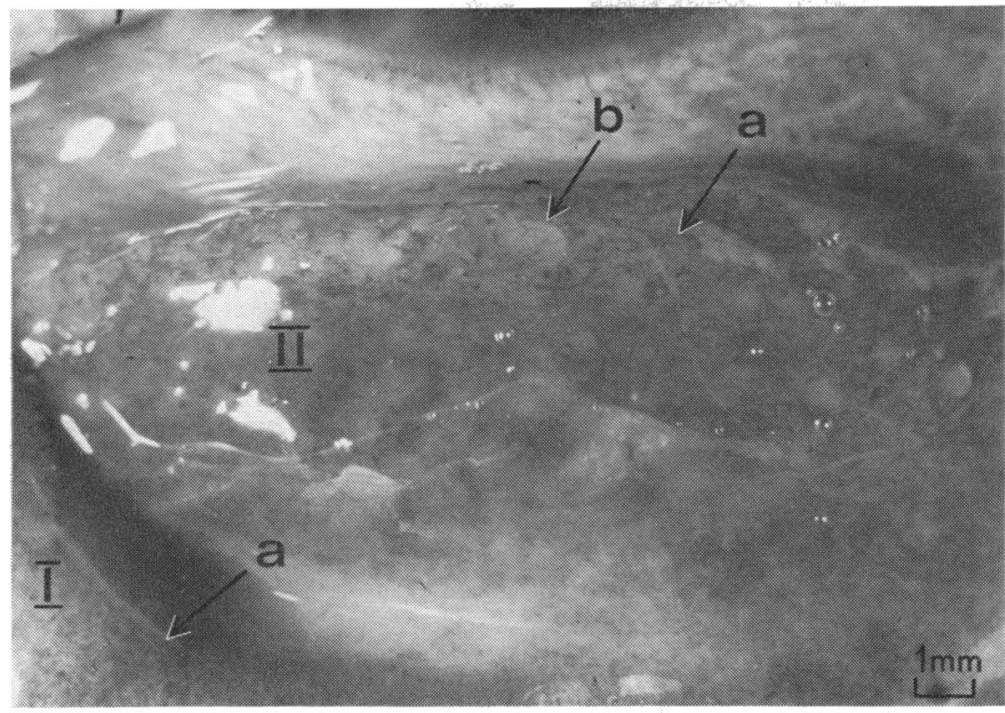

Fig. 7 Mixed papillary (a) and follicular reaction (b) with oedema and exudate in the lower lid conjunctiva in (I) tarsal and (II) fornix areas during the second week of type 19 adenovirus conjunctivitis

developed moderate to severe subepithelial punctate keratitis (Jones, 1962); most of these opacities were coarse in nature and visible with the naked eye (Fig. 14). Nearly half of these patients showed more than 15 to 20 lesions in the cornea, mainly within the interpalpebral fissure. The average time from onset of symptoms to the occurrence of subepithelial lesions was 17 days (10 to 35 days). The subepithelial keratitis persisted in the majority of these cases longer than a year.

DIFFERENTIAL CYTOLOGY

Conjunctival impressions taken in the early stage of keratoconjunctivitis showed a predominantly mononuclear cell exudate in two-thirds of the patients. In the remaining third there was a mixed mononuclear and polymorphonuclear cell response.

\section{ISOLATION OF VIRUS}

Adeno 19 was isolated in HEK cells from each of the 21 cases, with CPE appearing three to 19 days after inoculation. The interval between onset of the disease and collection of conjunctival specimens was less than one week in nine cases, one to two weeks in six cases, three to four weeks in five cases, and in one case it was 12 months.

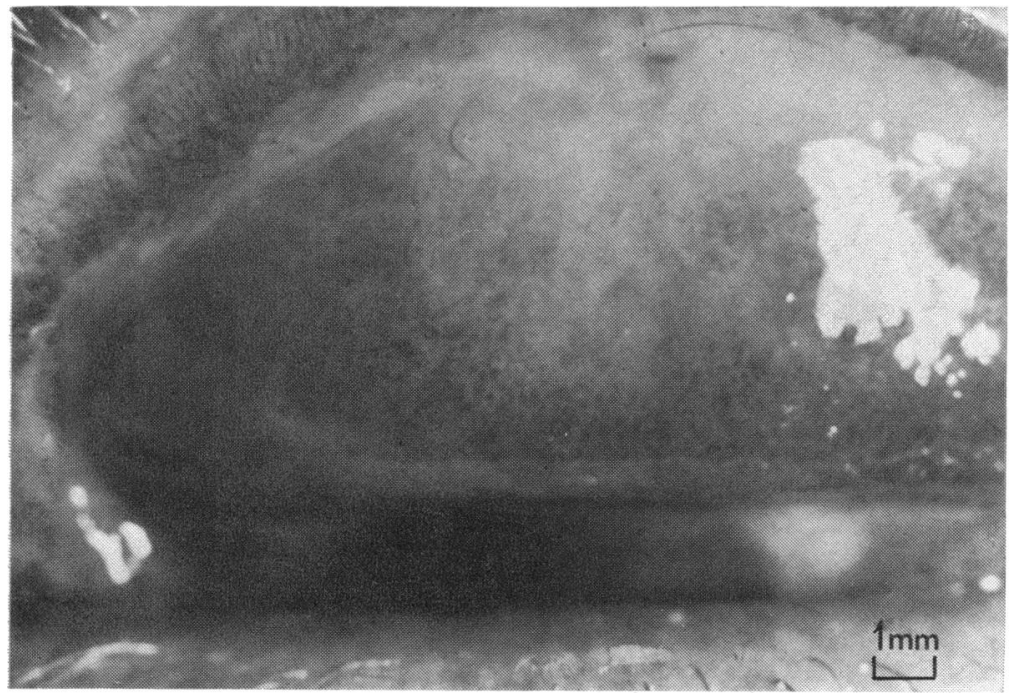

Fig. 8 Pseudomembrane in upper tarsal conjunctiva in the second week of type 19 adenovirus conjunctivitis 


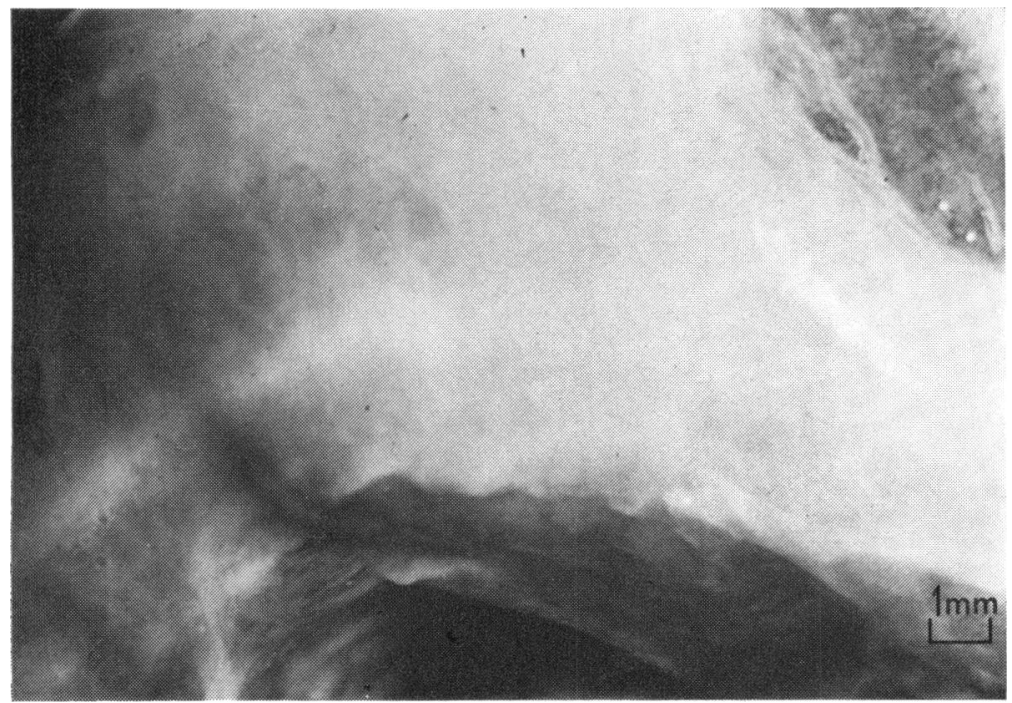

Fig. 9 Severe pseudomembrane in upper fornix area of conjunctiva in the second week of infection
Aliquots of the conjunctival specimens collected from nine patients were inoculated on to HEp2 cells in parallel with HEK cells. All the cultures were positive for adeno 19 in both systems, but in HEp2 cells the CPE appeared approximately 10 days later than in HEK cells.

\section{COMPLEMENT FIXATION TEST}

Paired or triplicate sera were tested in 15 patients. A minimum four-fold rise in titre was shown in eight patients. A high titre of $1 / 80$ to $1 / 160$ was obtained in six cases.

\section{TREATMENT}

While the aetiology was being determined, all patients were initially treated with eye drops or eye ointment of either chloramphenicol or neomycin to prevent secondary bacterial infection.

In nine patients with a mild or moderate keratoconjunctivitis treatment was continued with the antibiotics. The symptoms in these patients disappeared in two to four weeks, and the average course of the disease was approximately six weeks. The other 12 cases who were suffering from a more severe keratoconjunctivitis and had not benefited

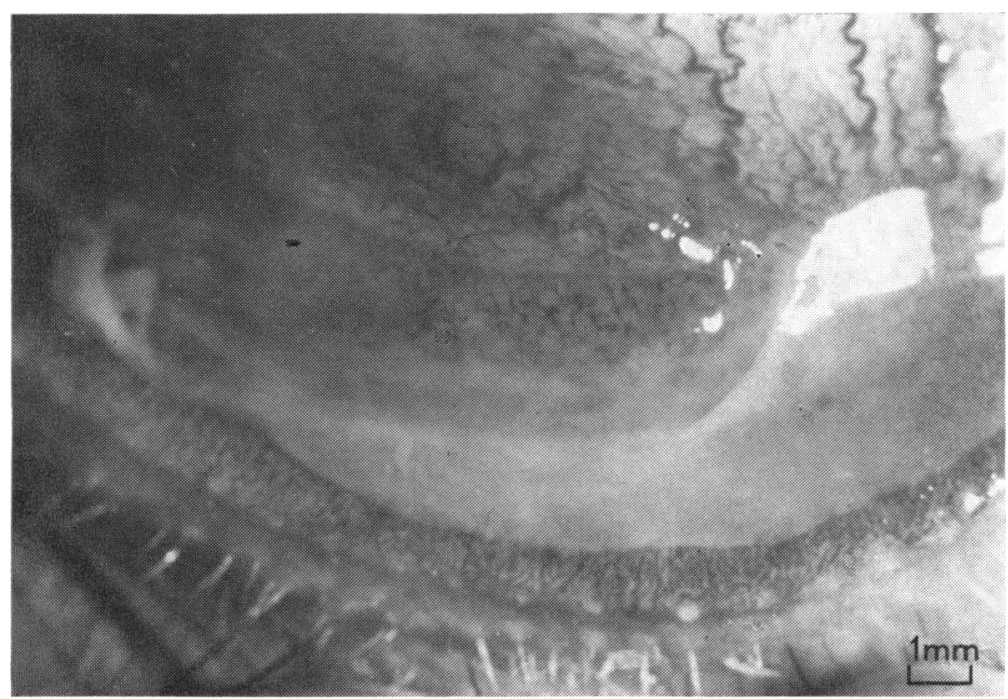

Fig. 10 Pseudomembrane almost covering lower lid conjunctiva in the second week of infection 


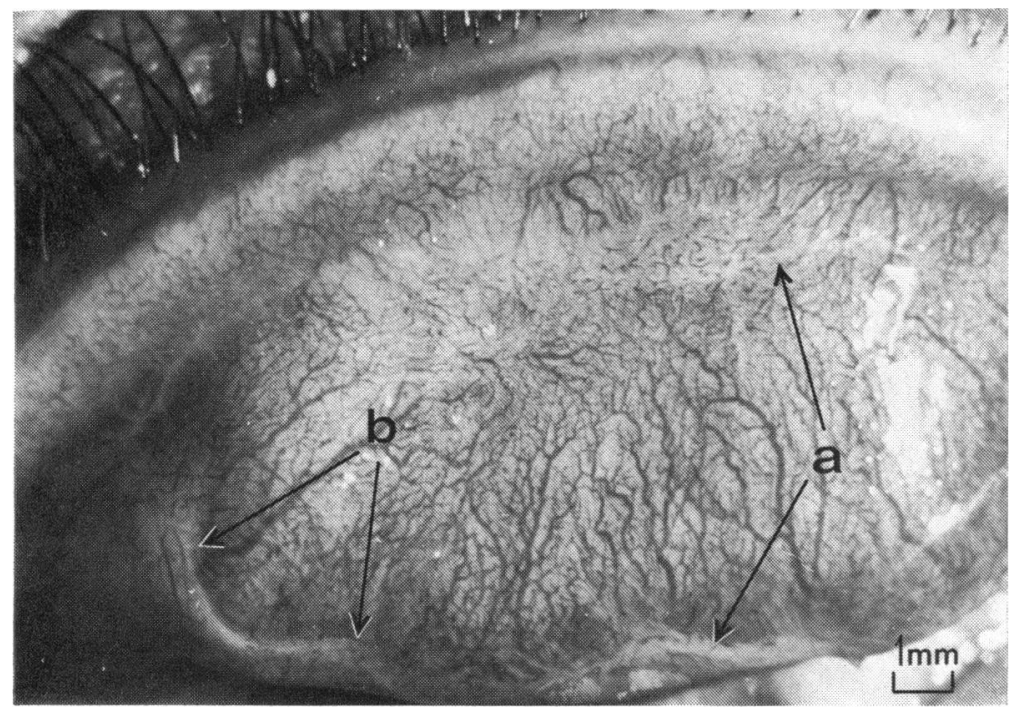

Fig. 11 Linear (a) and synechial scar (b) in upper tarsal conjunctiva four weeks after infection

from the antibiotic therapy were treated with a sevenday course of $1 \%$ trifluorothymidine eye-drops (TFT) hourly for the first day, two-hourly for the second day, and five times a day for the next five days. In this group of patients symptoms reduced in less than one to two days and had completely disappeared in two to four days. The range of duration of keratoconjunctivitis in this group of patients was three to four weeks.

\section{Discussion}

Keratoconjunctivitis Canada, and Scotland (Hierholzer et al., 1974; Keratoconjunctivitis due to adeno types 2, 3, 4, 5, Vas et al., 1974; Desmyter et al., 1974; Bell and

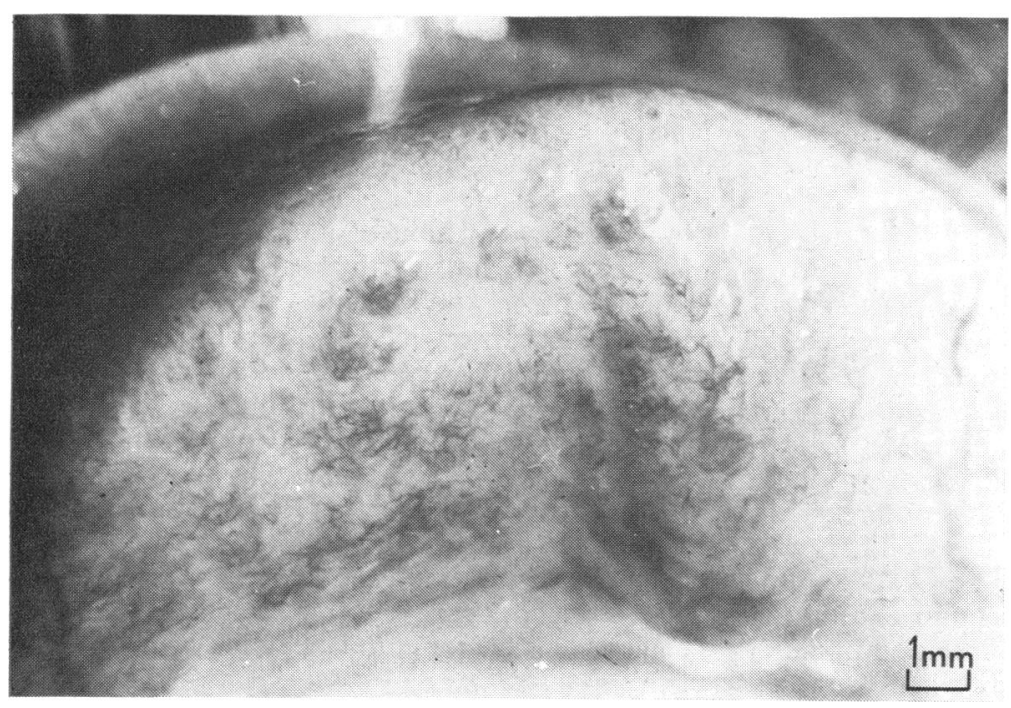

Fig. 12 Severe diffuse scarring in upper fornix conjunctiva following a severe pseudomembrane (fourth week) 


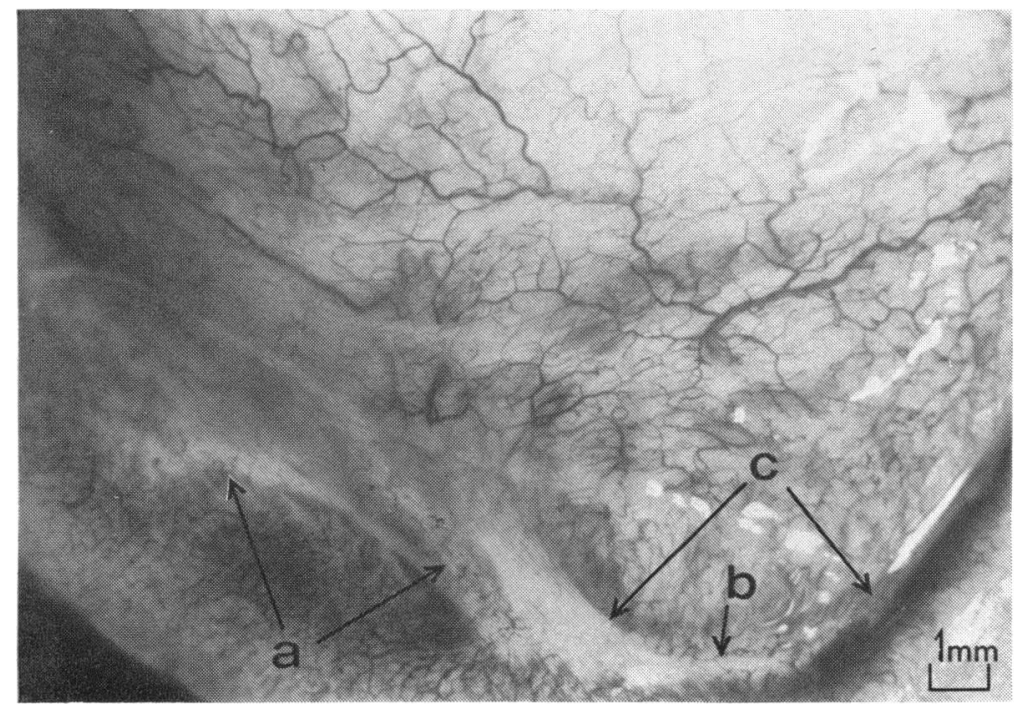

Fig. 13 Diffuse (a), linear (b), and severe synechial scarring (c) in lower lid conjunctiva (fourth week) with deformity of the lid

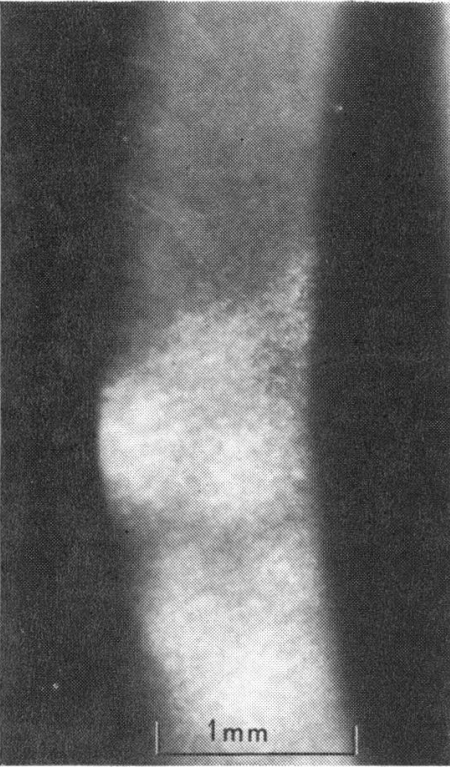

Fig. 14

Macrophotograph $(\times 20)$ of confluent coarse subepithelial punctate corneal opacities in the broad slit-lamp beam (Photograph by N. Brown)

Winton, 1975). The first proved case of adeno 19 keratoconjunctivitis in London was observed in June 1973; more cases were identified in the latter part of 1973 and in 1974, but there was no obvious seasonal variation in incidence of acute keratoconjunctivitis presenting in our clinic, nor was there any evidence of major community outbreaks in London during this period.

The simultaneous occurrence of adeno 19 keratoconjunctivitis in several different countries after the 18-year interval since the original isolation in Saudi Arabia has not been satisfactorily explained. It may have been due to the emergence of a pathogenic variant or to the arrival of the agent in areas in which the resulting disease would come under intensive study (Desmyter et al., 1974).

The clinical features of adeno 19 keratoconjunctivitis-a severe follicular conjunctivitis, sometimes with pseudomembrane and scarring, and commonly with a major subepithelial punctate keratitis but with little in the way of systemic disease-are all closely similar to those of epidemic keratoconjunctivitis caused by adeno 8 (Jawetz et al., 1955; Jones, 1962; Dawson et al., 1970; Hart et al., 1972). The ability of both agents to cause hospital outbreaks (Hierholzer et al., 1974; Vas et al., 1974) greatly accentuates this similarity and makes it clear that adeno 19 can cause epidemic keratoconjunctivitis.

The case of recurrent chronic papillary conjunctivitis that persisted for 16 months after an acute onset indicates the probable way in which iatrogenic hospital- or clinic-based outbreaks of eye-to-eye transmission of infection with adeno 19 may arise. It is noteworthy that infective virus was being shed from the patient's eyes after 12 months. of recrudescent or recurrent illness, the clinical features of which were entirely different from those of the classical case of acute follicular adenovirus eye infection with recovery within four to six weeks. Such a patient presenting to an ophthalmologist would hitherto not have aroused the suspicion of adenovirus infection, for even those trained especially in external eye diseases would have dismissed the possibility of adenovirus infection being responsible for chronic papillary conjunctivitis of this nature. 
It is easy to see how such a patient, with a rather nondescript illness, which in this case was referred as a probable case of conjunctivitis artefacta, would have a number of investigations carried out, including lachrymal syringing, and would not be segregated from other patients or be handled with particular care to prevent cross-infection. Such a case would therefore be especially likely to remain unrecognised and to supply the source of virus for a new outbreak of epidemic keratoconjunctivitis. It is therefore of importance that chronic or recurrent cases of papillary conjunctivitis should rouse the suspicion of adenovirus infection and should be appropriately investigated.

It is of interest that in this case the signs and symptoms of disease rapidly resolved, and eye cultures became negative for adenovirus on treatment with $1 \%$ TFT eye-drops hourly for the first day, two-hourly for the second day, and five times daily for the next five days. We had a clinical impression that this treatment also benefited and shortened the duration of illness in $\mathbf{1 2}$ cases in the present outbreak in which symptoms were severe and persistent. This is in contrast with the apparent ineffectiveness of $1 \%$ TFT drops in the ordinary run of sporadic adenovirus eye infection due to types other than 19 (Darougar, Hunter, Gibson, and Jones, in preparation).

While the number of specimens inoculated into HEp2 cells in this study was small, it appears that the sensitivity of this cell line is similar to that of HEK cells for the isolation of adeno 19, although the development of CPE was markedly slower. Other cell cultures, including primary human thyroid cells, human amnion cells, and primary African green monkey kidney cells, have also been satisfactory for the isolation of adeno 19 (Desmyter et al., 1974; Bell and Winton, 1975).

The authors are indebted to colleagues at Moorfields Eye Hospital for referring patients, to members of the External Eye Disease Clinic, Moorfields Eye Hospital, for the collection of clinical specimens, to Mr T. Tarrant for the graphics, and to the audiovisual department of the Institute of Ophthalmology for help in preparation of photographs. This work was supported by a locally organised clinical research grant, Moorfields Eye Hospital, City Road.

\section{References}

Bell, S. D., Jnr, Rota, T. R., and McComb, D. E. (1960). Adenovirus isolated from Saudi Arabia-six new serotypes. American Journal of Tropical Medicine and Hygiene, 9, 523-526.

Bell, E. J., and Winton, F. W. (1975). Keratoconjunctivitis caused by adenovirus type 19. British Medical Journal, 1, 91.

Communicable Disease Report/VS 74/41-44, 5 Oct-11 Nov. 1974.

Darougar, S., and Jones, B. R. (1971). Conjunctival swabbing for the isolation of TRIC agent (Chlamydia). British Journal of Ophthalmology, 55, 585-590.

Dawson, C. R., et al. (1970). Adenovirus type 8 keratoconjunctivitis in the United States. American Journal of Ophthalmology, 69, 473-480.

Dawson, C. R., Jones, B. R., and Darougar, S. (1975). Blinding and non-blinding trachoma-assessment of upper tarsal inflammatory disease and disabling lesions. Bulletin of the World Health Organisation, 52, 279-282.

Desmyter, J., et al. (1974). Keratoconjunctivitis caused by adenovirus type 19. British Medical Journal, 4, 406.

Hart, J. C. D., et al. (1972). Epidemic keratoconjunctivitis -a virological and clinical study. Transactions of the Ophthalmological Society of the U.K., 92, 795-801.

Hierholzer, J. C., et al. (1974). Adenovirus type 19 keratoconjunctivitis. New England Journal of Medicine, 290, 1436.

Jawetz, E., et al. (1955). Studies on the etiology of epidemic keratoconjunctivitis. American Journal of Ophthalmology, 40, 200-211.

Jones, B. R. (1962). Adenovirus infections of the eye in London. Transactions of the Ophthalmological Societies of the U.K., 82, 621-644.

McSwiggan, D. A., et al. (1975). Comparison of the sensitivity of human embryo kidney cells, HeLa cells, and W138 cells for the primary isolation of viruses from the eye. Journal of Clinical Pathology, 28, 410-413.

Vas, S. I., et al. (1974). Keratoconjunctivitis due to adenovirus type 19-Canada. Morbidity and Mortality, 23, 185186. 